\title{
Graphic Dependences for Determination of Temperature and Electric Field Strength in Arc Discharge
}

\author{
Evgeniy N. Vasil'ev* \\ Institute of Computational Modeling SB RAS \\ 50/44 Academgorodok, Krasnoyarsk, 660036, Russia
}

Received 17.12.2014, received in revised form 01.04.2015, accepted 19.09.2015

The dependences of the temperature $T$ and electric field strength $E$ on the current $I$ and radius $R$ for a axisymmetric stationary equilibrium arc discharge in air, argon, carbon dioxide and hydrogen under atmospheric pressure are determined from the solution to the Elenbaas-Heller equation. The results of computations are given on diagrams in the plane lgI-R in the form of lines of constant values. The diagrams make it possible to rapidly estimate parameters of the arc discharge for the given values of I and $R$.

Keywords: the arc discharge, temperature, electric field strength, power balance.

DOI: 10.17516/1999-494X-2015-8-7-901-907.

\section{Графические зависимости}

\section{для определения температуры и напряженности}

\section{электрического поля в дуговом разряде}

Е.Н. Васильев

Институт вычислительного моделирования СО РАН Россия, 660036, Красноярск, Академгородок, 50/44

На основе решения уравнения Эленбааса-Хеллера рассчитаны зависимости температуры $T$ u напряженности электрического поля $E$ от радиуса $R$ и силь тока I для осесимметричного стационарного равновесного дугового разряда в воздухе, аргоне, углекислом газе и водороде при атмосферном давлении. Результаты расчетов представлены на диаграммах в плоскости $\lg I-R$ в виде линий постоянных значений Т и Е. Диаграммы позволяют оперативно оценивать параметры дугового разряда для заданных значений $R$ и I.

Ключевые слова: дуговой разряд, температура, напряженность электрического поля, энергетический баланс.

(C) Siberian Federal University. All rights reserved

* Corresponding author E-mail address: ven@icm.krasn.ru 


\section{Введение}

При разработке и оптимизации новых электродуговых устройств и технологий необходима информация об энергетическом состоянии разрядной плазмы. Экспериментальное исследование и измерение энергетических параметров требует очень значительных материальных и временных затрат. Расчетное определение параметров разрядов проводится с помощью вычислительных моделей различной степени сложности, основанных на совместном численном решении систем уравнений газовой динамики и электродинамики [1-4]. Такие задачи, как правило, являются ресурсоемкими, требуют мощных вычислительных средств и больших временных затрат на разработку и тестирование. По этим причинам остается актуальным применение более простых одномерных математических моделей, позволяющих оперативно рассчитывать основные параметры дуговых разрядов. С помощью такой математической модели на основе уравнения Эленбааса-Хеллера были исследованы особенности формирования дугового разряда в теплопроводном и радиационных режимах $[5,6]$. Также были рассчитаны и построены на плоскости $\lg I-R$ изолинии для заданных характерных соотношений интегральных и удельных мощностей механизмов теплопроводности и излучения в разряде и определены соответствующие им зависимости температуры и напряженности электрического поля для различных газов [7-9]. Эти зависимости, прежде всего, устанавливали границы диапазонов влияния и доминирования энергетических механизмов и давали возможность определять значения параметров разряда, соответствующие этим граничным состояниям. Однако для промежуточных энергетических состояний, особенно вдали от границ, проводить оценку значений параметров разряда с помощью зависимостей для граничных состояний достаточно сложно. Графические зависимости, представленные в данной работе, дают возможность определения температуры и напряженности электрического поля с меньшей погрешностью в более широком диапазоне значений радиуса и силы тока.

\section{Постановка задачи}

Для расчета распределения температуры в осесимметричном разряде использовано уравнение Эленбааса-Хеллера, описывающее баланс энергетических механизмов джоулевой диссипации, теплопроводности и излучения

$$
\frac{1}{r} \frac{\partial}{\partial r}\left(r \lambda \frac{\partial T}{\partial r}\right)+q_{J}-q_{R}=0
$$

где $r$ - радиус; $T$ - температура; $\lambda$ - коэффициент теплопроводности газа; $q_{J}=\sigma E^{2}-$ мощность джоулевой диссипации; $q_{R}-$ объемная мощность радиационных энергопотерь. Уравнение (1) дополняется граничными условиями в центре разрядной области и на внешней границе

$$
\left[\frac{\partial T}{\partial r}\right]_{r=0}=0,[T]_{r=R}=T_{0}
$$

Напряженность электрического поля $E$ зависит от силы тока и распределения электропроводности $\sigma(r)$ по радиусу: 


$$
E=I / 2 \pi \int_{0}^{R} \sigma(r) r d r
$$

Электрические дуги при поперечном размере высокотемпературной зоны порядка 1 см имеют малую оптическую толщину, поэтому для расчета величины $q_{R}$ было использовано приближение объемного излучателя [5].

Решение краевой задачи (1) - (3) проводилось численно с учетом температурных зависимостей свойств газов, которые вводились в программу в виде таблиц для степеней черноты $\varepsilon(T)$, коэффициентов электропроводности $\sigma(T)$ и теплопроводности $\lambda(T)$. Коэффициенты черноты воздуха взяты в [10,11], аргона в [12], углекислого газа в [11], водорода в [13]. Транспортные свойства воздуха в [14], аргона в [15], углекислого газа в [16], водорода в [17, 18].

\section{Результаты расчетов}

Вычислительная модель позволяет рассчитать структуру и параметры разряда для разных газов при различных значениях силы тока $I$ и радиуса расчетной области $R$. Из решения уравнений (1)-(2) определяется распределение температуры и электропроводности, с помощью формулы (3) рассчитывается напряженность электрического поля $E$. Для проверки адекватности вычислительной модели проведено сравнение расчетных и экспериментальных данных. На рис. 1 приведены результаты измерений температурного распределения в дуговом разряде, горящем в углекислом газе [19]. Температурный профиль дуги определяли несколькими спектроскопическими методами, ошибки в измерении температуры составляли 6-7 \%. На график с обобщенным экспериментальным профилем (кривая 1) наложено расчетное распределение температуры (кривая 2). Расчетное значение температуры на оси разряда $T_{\max }=10,54$ кК и отличается от измеренного примерно на $4 \%$, в периферийной части разряда разница увеличивается, по-видимому, из-за влияния обдува дуги, необходимого для ее пространственной стабилизации. Такое отличие в значениях $T_{\max }$ находится в пределах ошибки



Рис. 1. Радиальное распределение температуры в разряде при $R=1$ мм

$$
-903-
$$


измерений температуры и погрешности расчета теплофизических свойств газов, которая, по мнению авторов работ [10-18], составляет от нескольких процентов до 20-30 \%. Данный расчет структуры разряда был проведен при величинах пространственного шага в 0,05 и 0,025 мм, при этом различие в значении $T_{\max }$ для этих двух вариантов было зафиксировано незначительным $(\approx 0,02 \%)$.

Важнейшими характеристиками дугового разряда являются температура и напряженность электрического поля. От величины температуры в столбе разряда зависят переносные свойства плазмы и интенсивность взаимодействия с электромагнитным полем, а напряженность электрического поля определяет мощность, необходимую для компенсации энергопотерь и поддержания разряда. Для определения характеристик разряда были проведены расчеты в достаточно широком диапазоне значений радиуса $R=2 \div 40$ мм и силы тока $I=1 \div 10000 \mathrm{~A}$. На основе расчетов построены в виде изолиний графические зависимости $T_{\max }$ и $E$ на плоскости $\lg I-R$ для разряда в воздухе, аргоне, углекислом газе и водороде при атмосферном давлении. Значения на изолиниях $T_{\max }$ приведены в кК, на изолиниях $E$ в размерности кВ/м. Расчеты проведены при величине пространственного шага 0,5 мм, при необходимости величина шага снижалась до 0,1 мм.

Графические зависимости для воздуха представлены на рис. 2. Изолинии максимальной температуры в центре разряда $T_{\max }$ отображены с шагом 0,5 кК (рис. $2 a$ ). Поскольку на рис. 2 изолинии расположены достаточно близко друг к другу, значения температуры в кК даны только для части изолиний, имеющих увеличенную толщину. Изолинии напряженности электрического поля $E$ построены с переменным шагом (рис. 2б). При малых значениях $E$ изолинии имеют две ветви, которые имеют угол наклона противоположного знака. Ветви соответствуют разным режимам теплоотвода из столба разряда: нижние расположены к области, где преобладает теплопроводный механизм, верхние лежат в зоне доминирования излучения. В месте перегиба изолиний влияние теплопроводного и радиационного механизмов в энергобалансе разряда является соизмеримым.

На рис. 3 приведены характеристики дугового разряда в аргоне. Изолинии максимальной температуры $T_{\max }$ также отображены с шагом 0,5 кК, значения на изолиниях даны с шагом 1 кК

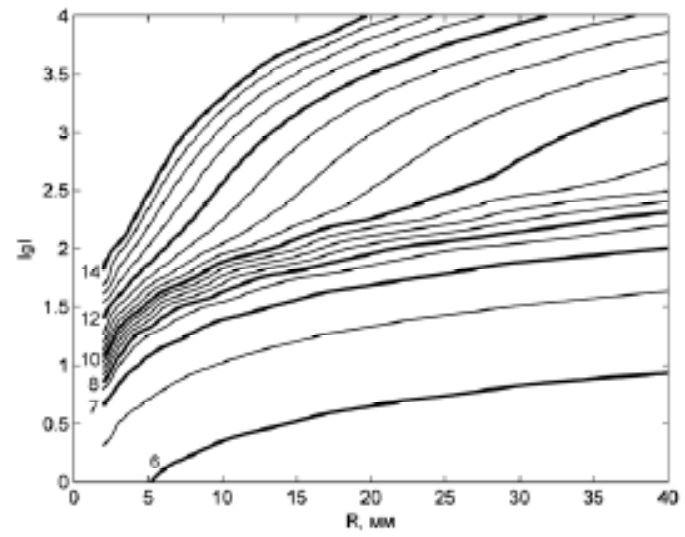

a)

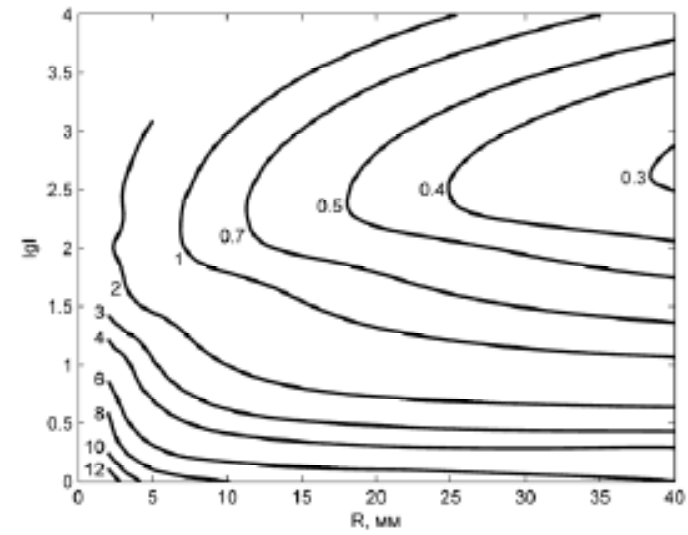

б)

Рис. 2. Изолинии температуры (а) и напряженности электрического поля (б) для разряда в воздухе 
(рис. $3 a$ ). Изолинии напряженности электрического поля $E$ приведены с переменным шагом. При сравнении характеристик разряда в воздухе и аргоне для изолиний $T_{\max }$ в аргоне следует отметить более равномерное и упорядоченное расположение, а для напряженности электрического поля характерны существенно меньшие значения.

Особенностью графических зависимостей для разряда в углекислом газе является наличие достаточно протяженных горизонтальных участков изолиний $T_{\max }$ и $E$ в диапазоне больших значений радиуса и малых значений силы тока, которому соответствует правая нижняя часть плоскости $\lg I-R$ (рис. 4). На горизонтальных участках изолиний характеристики разряда не зависят от его радиуса. Данная особенность разряда в $\mathrm{CO}_{2}$ ранее была отмечена и исследована в [8], где была установлена ее причина - эффект экранирования теплопроводного механизма вблизи внешней границы разряда. Этот эффект обусловлен высокими значениями степени

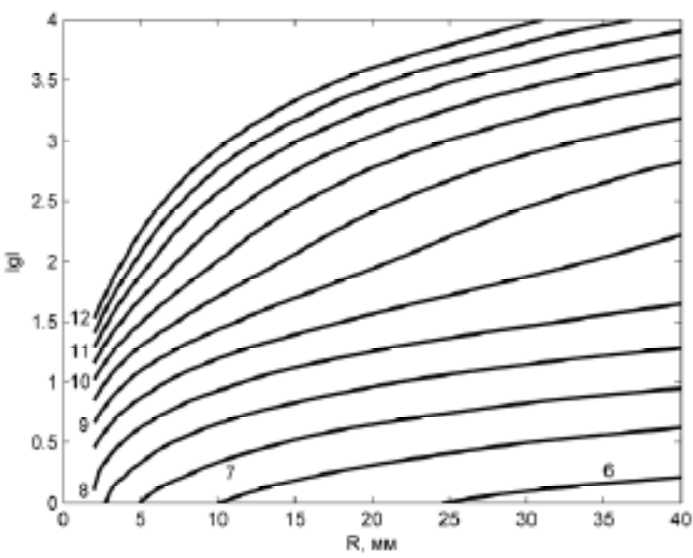

a)

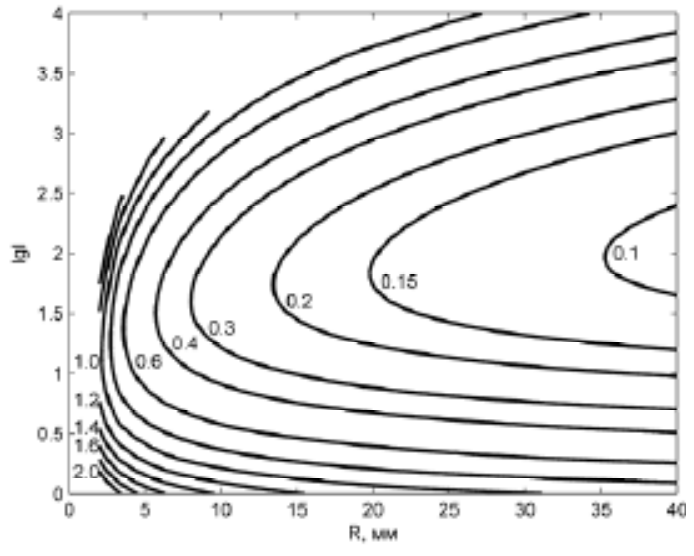

б)

Рис. 3. Изолинии температуры (а) и напряженности электрического поля (б) для разряда в аргоне

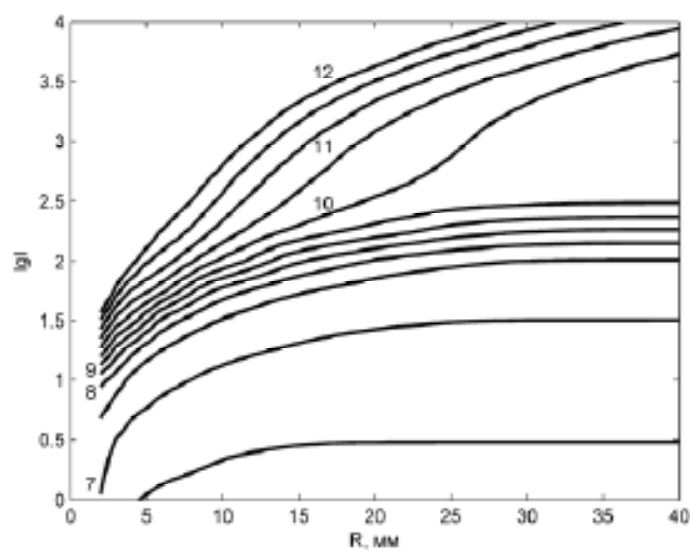

a)

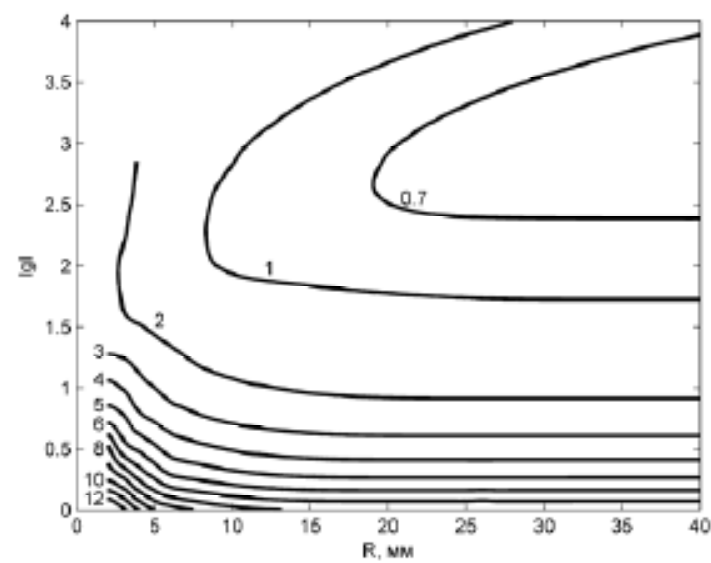

б)

Рис. 4. Изолинии температуры (а) и напряженности электрического поля (б) для разряда в углекислом газе

$$
-905-
$$




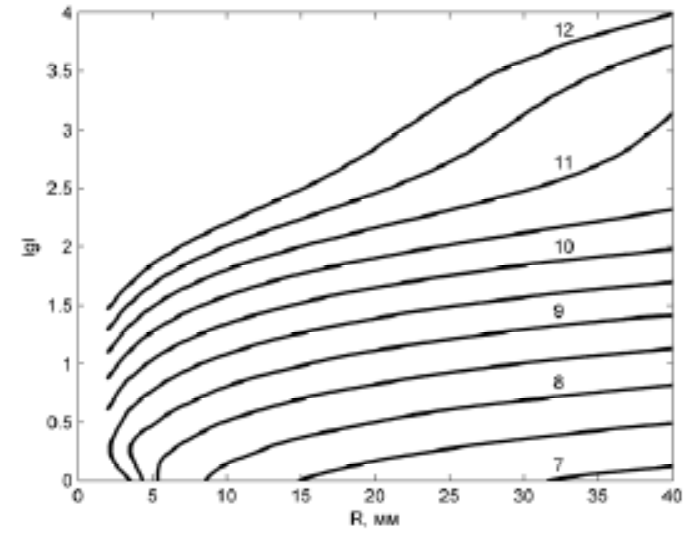

a)

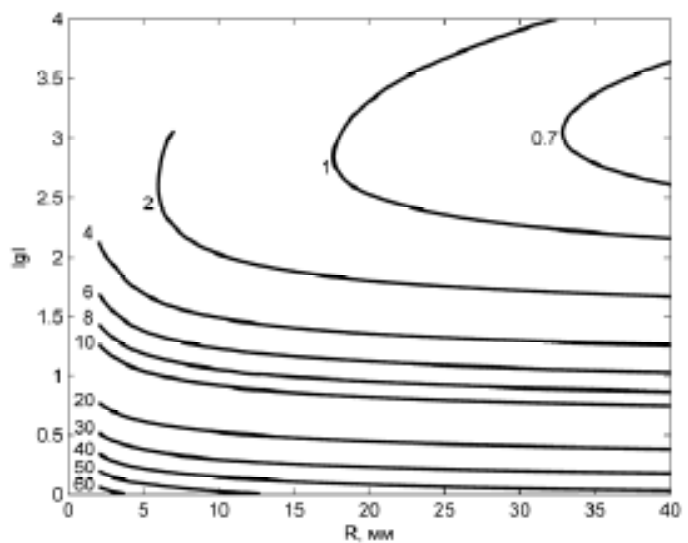

б)

Рис. 5. Изолинии температуры (а) и напряженности электрического поля (б) для разряда в водороде

черноты $\mathrm{CO}_{2}$ в диапазоне низких температур (1-2 кК), при этом периферийный слой газа с такой температурой, имеющий высокую излучающую способность, отводит всю подводимую от центральной горячей зоны теплоту за пределы разряда путем радиационного механизма, фактически экранирует центральную высокотемпературную зону разряда от теплопроводного теплообмена с внешней стенкой и тем самым обеспечивает пространственную локализацию столба разряда.

Характеристики дугового разряда в водороде отображены на рис. 5. Отличительной особенностью разряда в водороде является существенно более высокие значения напряженности электрического поля, особенно в области малых значений силы тока. Это обусловлено большим значением коэффициента теплопроводности. Так, зависимость $\lambda(T)$ для водорода имеет локальный максимум $\lambda_{\max }=15.1 \mathrm{BT} /(\mathrm{M} \cdot \mathrm{K})$ при $4 \cdot 10^{3} \mathrm{~K}$, а значения $\lambda_{\max }$ для воздуха и углекислого газа находятся на уровне $4 \mathrm{BT} /(\mathrm{м} \cdot \mathrm{K})$ при $T=7 \cdot 10^{3} \mathrm{~K}$. Таким образом, повышенная мощность теплопроводных энергопотерь для разряда в водороде компенсируется за счет большей напряженности электрического поля.

\section{Заключение}

В работе представлены в виде диаграмм на плоскости $\lg I-R$ характеристики равновесной электродуговой плазмы для некоторых технически важных газов. Графические зависимости для гелия были приведены в [9]. Демонстрируемые графические зависимости дают общую картину энергетического состояния электродуговой плазмы и позволяют оперативно оценивать температуру, напряженность электрического поля и энергетические затраты в осесимметричном стационарном дуговом разряде, горящем в воздухе, аргоне, углекислом газе и водороде при атмосферном давлении, в широком диапазоне значений радиуса и силы тока.

\section{Список литературы}

[1] Васильев Е.Н., Деревянко В.А., Славин В.С. // Теплофизика высоких температур. 1986. T. 24. № 5. C. 844. 
[2] Васильев Е.Н., Нестеров Д.А. // Вестник Красноярского государственного университета. 2004. № 5. С. 104.

[3] Васильев Е.Н., Нестеров Д.А. // Теплофизика высоких температур. 2005. Т. 43. № 3. C. 401.

[4] Васильев Е.Н., Нестеров Д.А. // Теплофизика высоких температур. 2007. Т. 45. № 2. C. 165.

[5] Васильев Е.Н. // Теплофизика и аэромеханика. 2010. Т. 17. № 3. С. 441.

[6] Васильев E.Н. // Журнал Сибирского федерального университета. Техника и технологии. 2013 (6). № 2.183.

[7] Васильев Е.Н. // Журнал технической физики. 2012. Т. 82. № 12. С. 47.

[8] Васильев Е.Н. // Журнал технической физики. 2013. Т. 83. № 10. С. 26.

[9] Васильев Е.Н. // Журнал технической физики. 2014. Т. 84. № 12. С. 38.

[10] Авилова И.В., Биберман Л.М., Воробьев В.С. и др. Оптические свойства горячего воздуха. М.: Наука, 1970. 320 с.

[11] Каменщиков В.А., Пластинин Ю.А., Николаев В.М., Новичкий Л.А. Радиационные свойства газов при высоких температурах. М.: Машиностроение, 1971. 440 с.

[12] Ветлуиякий В.Н., Онуфриев А.Т., Севастьяненко В.Г. // Журнал прикладной механики и технической физики. 1965. № 4. С. 71.

[13] Москвин Ю.В. // Теплофизика высоких температур. 1968. Т. 6. № 1. С.1.

[14] Соколова И.А. // Журнал прикладной механики и технической физики. 1973. № 2. C. 80 .

[15] Devoto R.S. // Physics of Fluids. 1967. V. 10. № 2. P. 354.

[16] Андриатис А.В., Соколова И.А. // Математическое моделирование. 1995. Т. 7. № 2. C. 89 .

[17] Devoto R.S. // Journal of Plasma Physics. 1968. V. 2. № 4. P. 617.

[18] Копански Ю. // Теплофизика высоких температур. 1973. Т. 11. № 3. С. 644.

[19] Киселевский Л.И., Соловьянчик Д.А., Фещенко 3.П. // Журнал прикладной спектроскопии. 1972. Т. 16. № 2. С. 234. 